Document downloaded from:

http://hdl.handle.net/10251/40394

This paper must be cited as:

Benlloch-Dualde, JV.; Buendía García, F.; Juan-Carlos Cano; Blanc Clavero, S.; Lemus Zúñiga, LG. (2013). ¿Cómo ayudar a los docentes a crear entornos de aprendizaje dinámicos mediante el uso de la tinta digital?. TEXTOS de la CiberSociedad. 17(1):85-98.

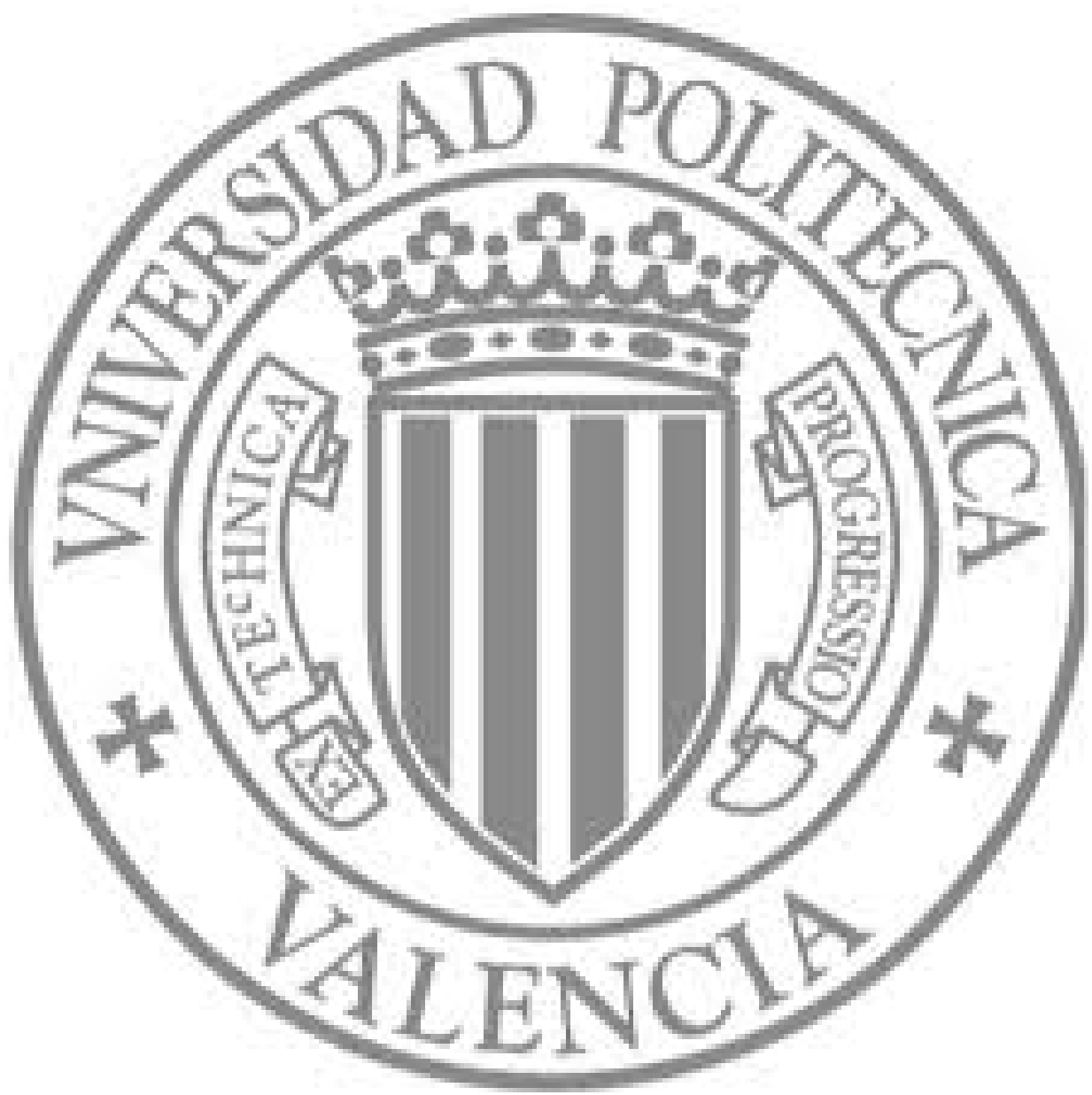

The final publication is available at

Copyright

Observatorio para la CiberSociedad 


\title{
¿Cómo ayudar a los docentes a crear entornos de aprendizaje dinámicos mediante el uso de la tinta digital?
}

\author{
Jose-Vicente Benlloch-Dualde, Universitat Politècnica de València, \\ España \\ Félix Buendía, Universitat Politècnica de València, España \\ Juan-Carlos Cano, Universitat Politècnica de València, España \\ Sara Blanc, Universitat Politècnica de València, España \\ Lenin Lemus, Universitat Politècnica de València, España
}

\begin{abstract}
Resumen: Existen evidencias de que las Tecnologias de Tinta Digital (TTD) pueden facilitar cambios importantes que permitan abordar los típicos problemas asociados a los modelos pedagógicos basados en la lección magistral. En el contexto de los estudios universitarios de Informática, existe un consenso bastante generalizado del potencial de las TTD, y de las tabletas PC en particular, como instrumentos que fomentan la interacción en el aula y promueven entornos de aprendizaje mucho más dinámicos. Teniendo en cuenta la complejidad de las tecnologías y su explosivo desarrollo, resulta conveniente apoyar a los docentes que planean incorporarlas, mediante la conceptualización de sus posibilidades educativas. Con este fin, el presente trabajo analiza la utilización de mapas conceptuales como herramientas para modelizar tanto el dominio instructivo como el dominio tecnológico. Para validar el enfoque propuesto se han desarrollado una serie de talleres, dirigidos a profesores de ingeniería, donde además de presentar el potencial educativo de estas tecnologías, se les permite expeUmentar con tabletas PCy otros dispositivos de tinta digital. Antes de comenzar, los asistentes responden a un pre-cuestionario que trata de recoger los requisitos instructivos del contexto de cada profesor, cuyas preguntas se han generado a partir de un mapa conceptual instructivo general. En segundo lugar, a partir del mapa conceptual de las TTD, se han generado unas recomendaciones de uso que se han convertido en cuestiones para que el docente confirme, al final del taller (post-cuestionario), su grado de acuerdo. Entonces, se buscan las relaciones entre las respuestas de ambos cuestionarios, así como el grado de concordancia con las recomendaciones generadas. Más de setenta profesores pertenecientes a diferentes departamentos han participado en estos talleres y la evaluación general es muy favorable.
\end{abstract}

Palabras Clave: Tinta Digital, Tableta PC, Pen-based Technology, Mapas Conceptuales, Diseño Instructivo

Abstract: There is evidence that Pen-Based Technologies (PBTs) can facilitate radical changes that would make it possible to address problems typically associated with lecture-based pedagogical models. In the context of university-level Computer Science studies, there is widespread consensus that PBTs - and tablet PCs, in particular - are tools that have great potential for encouraging interaction in the classroom and promoting a far more dynamic learning environment. In view off the complexity of these types of technology and their explosive evolution, it would be advisable to assist teachers who wish to utilize them by developing strategies based on a conceptualization of their edu-

TEXTOS. Revista Internacional de Aprendizaje y Cibersociedad

Volumen 17, Número 1, 2013, http://aprendizaje-cibersociedad.com/journal, ISSN 1577-3760

(C) Common Ground, Jose-Vicente Benlloch-Dualde, Felix Buendia, Juan-Carlos Cano, Sara Blanc, Lenin

Lemus, Todos los Derechos Reservados, Permisos: cg-support@commongroundpublishing.com 
cational capabilities. With this objective in mind, this article analyzes the use of concept maps as tools for modeling both the instructional domain and the PBTs domain. To validate the proposed approach, a number of workshops were conducted, in which professors of Engineering were given a presentation on the educational capabilities of these technologies and then had the opportunity to experiment with tablet PCs and other digital ink devices. Participants completed a pre-questionnaire at the start of the workshop; its questions were generated from a generic concept map for the instructional domain, and its purpose was to list each professor's instructional requirements. Next, recommendations for use were developed, based on the PBTs concept map, and converted to the questions on a post-questionnaire completed at the end of the workshop, on which the teachers indicated the degree to which they agreed with the recommendations. More than 70 professors from different departments participated in these workshops, and their overall evaluation was quite favorable.

Keywords: Digital Ink, Tablet PC, Pen-based Technology, Concept Maps, Instructional Design

\section{Introducción}

T A INCORPORACIÓN GENERALIZADA de presentaciones electrónicas como apoyo a la lección magistral, representó hace unos años una innovación tecnológica frente a los métodos convencionales de uso de las transparencias o de la pizarra. Sin embargo, en muchos casos, este hecho ha significado la consolidación de un modelo didáctico centrado en el papel del profesor como transmisor del conocimiento.

En un informe publicado recientemente (Fundación Telefónica, 2011: 47), en relación al modelo de aprendizaje y enseñanza, se afirma que "se aprecia una tendencia hacia un modelo participativo y colaborativo en el que el aprendizaje se produce cuando el alumno desarrolla sus actividades y adquiere el conocimiento a través de la interacción con el entorno". El informe va un poco más allá al manifestar que "se prevé, por ejemplo, que en el año 2015 el $80 \%$ de los profesores universitarios aplicarán nuevos modelos didácticos con apoyos TIC en sus clases". En otro informe (Fundación de la Innovación Bankinter, 2011: 10-17) elaborado en el marco del proyecto Future Trends Forum, se propone un decálogo para contribuir a una reforma profunda en el terreno educativo. Uno de los diez principios planteados lo denominan Educación 2.0 y lo relacionan con el empleo de las TIC para mejorar la calidad de la enseñanza y el aprendizaje, apoyando tanto a alumnos como a profesores en la introducción de métodos innovadores que conformen una educación más atractiva y eficaz.

Numerosos estudios evidencian que las TIC pueden aportar importantes cambios en el aula, reduciendo algunos de los problemas asociados al modelo tradicional de enseñanza centrado en el profesor (Sneller, 2007). Sin embargo, la simple incorporación de la tecnología no garantiza una mejora en los logros de aprendizaje. Para conseguir una intervención exitosa se requieren unos criterios claros en el diseño e implantación de los entornos soportados por la tecnología. Tomando como base los conocidos "Seven Principles for Good Practice in Undergraduate Education" (Chickering y Gamson, 1987), encontramos distintos trabajos que proporcionan recomendaciones generales de cómo utilizar las tecnologías para enriquecer y ampliar, entre otras cosas, el contacto entre profesores y estudiantes, la cooperación entre estudiantes, el aprendizaje activo o la retroalimentación oportuna (Ehrmann, 2008).

Si es indudable que las posibilidades de un ordenador personal son múltiples, éstas se incrementan de forma significativa cuando incorporamos, como elemento de la interfaz de usuario, un bolígrafo especial que produce tinta digital, dando nombre a las llamadas "Pen- 
based Technologies" o Tecnologías de Tinta Digital (TTD). Entre los dispositivos de estas tecnologías destaca la tableta PC, que es un computador portátil convencional cuya pantalla actúa tanto como dispositivo de presentación como de entrada de datos, gracias a ese bolígrafo mencionado anteriormente. Estos dispositivos permiten realizar todo tipo de trazos a mano alzada para escribir, dibujar, realizar esquemas y diagramas, producir bocetos, expresar ideas de forma visual, todo ello de forma semejante a como se haría con un bolígrafo y un papel, pero con las ventajas añadidas de los formatos digitales.

Las características de las tabletas PC ayudan a desarrollar entornos educativos mucho más interactivos, al tiempo que permiten modificar el papel que tanto profesores como alumnos tienen en el aula. En particular, el potencial de estas tecnologías se incrementa notablemente cuando los equipos del profesor y de los estudiantes se conectan en red. $\mathrm{Si}$ a este hecho añadimos la utilización de las llamadas herramientas de presentación y colaboración, como Classroom Presenter ${ }^{1}$ o Dyknow ${ }^{2}$, se habilitan una serie de posibilidades educativas entre las que se podrían destacar las siguientes:

1. El profesor puede cargar una presentación electrónica y compartirla con sus estudiantes. Además, de forma espontánea, puede realizar anotaciones sobre la presentación original, que aparecen igualmente en los dispositivos de los estudiantes.

2. Los alumnos pueden, a su vez, realizar sus propias anotaciones sobre la instancia recibida en su equipo.

3. Los alumnos pueden enviar sus contribuciones al profesor que podrá previsualizarlas de modo privado en su equipo o mostrarlas, utilizando un cañón de proyección, a toda la clase para así fomentar la discusión. Del mismo modo, el profesor puede realizar anotaciones sobre el material mostrado y, si lo estima conveniente, difundirlo comentado a todos los alumnos.

4. Los profesores pueden plantear sondeos a sus alumnos con preguntas de tipo verdadero/falso o de respuesta múltiple que se pueden contestar seleccionando la opción correspondiente. Esta utilidad se asemeja a los diversos sistemas de respuesta de la audiencia, traducido del término inglés Classroom/Student/Audience Response Systems (Deal, 2007), más conocidos como “clickers". De la misma forma que en estos sistemas, el profesor puede recoger las respuestas y mostrar de forma gráfica las correspondientes estadísticas.

5. Los alumnos pueden trabajar en grupos y compartir determinados documentos que podrán presentar en público, una vez elaborados.

6. Profesores y alumnos pueden almacenar, en formato electrónico, toda la información generada en el aula, incluyendo las anotaciones en tinta digital y, por supuesto, publicarla en cualquier repositorio de la asignatura.

Dada la complejidad de las tecnologías anteriores y su rápido desarrollo, parece necesario establecer procedimientos que proporcionen guías o recomendaciones a los profesores que se plantean incorporar las TTD en un determinado contexto formativo. Los procedimientos propuestos se basan en el modelado previo de los distintos elementos de conocimiento que

\footnotetext{
${ }^{1}$ Classroom Presenter (http://www.cs.washington.edu/education/d1/presenter/).

${ }^{2}$ DyKnow (http://dyknow.com/products/vision).
} 
caracterizan, por un lado, el enfoque instructivo del curso o experiencia formativa y, por otro, las tecnologías que se va a incorporar, en nuestro caso, las TTD.

De entre los distintos enfoques que se pueden utilizar para organizar el conocimiento, se han seleccionado los mapas conceptuales (Novak, 2008) porque permiten representar distintos tipos de información, al tiempo que proporcionan un formato estructurado de gran flexibilidad y potencial. Aunque estas estructuras no proveen una formulación semántica en sentido estricto, pueden ser convertidas a otras notaciones formales que pueden ser procesadas de una forma sistemática. Por ejemplo, pueden ser un primer paso en la construcción de una ontología. Además, los mapas conceptuales pueden ser utilizados de forma flexible para representar diseños formativos específicos adaptados a entornos donde se incorporan las TIC (Buendía, 2011). Por último, otra ventaja de utilizar los mapas conceptuales sobre otras soluciones, es que resulta una estructura conocida para muchos profesores, que suelen utilizarlos y evaluarlos en distintos contextos formativos. En resumen, este trabajo analiza la conveniencia de modelizar los dominios instructivo y tecnológico mediante mapas conceptuales, como paso previo a la generación de guías o recomendaciones para diseñar e implantar entornos de aprendizaje más interactivos apoyados en el uso de tabletas PC.

El resto del artículo se organiza como sigue. La sección 2 repasa trabajos relacionados, haciendo un especial énfasis en aquéllos centrados en orientar a los profesores para diseñar cursos basados en el uso de tabletas PC. La sección 3 describe cómo se utilizan los mapas conceptuales para ayudar a los docentes en la incorporación de estas tecnologías a su entorno educativo. La sección 4 presenta un ejemplo concreto de las guías generadas en el contexto de una asignatura de informática industrial. La sección 5 presenta la evaluación de la propuesta y, por último, la sección 6 expone las conclusiones.

\section{Trabajos relacionados}

En los últimos años, se han sucedido las experiencias de uso de las tabletas PC en todos los niveles educativos, desde primaria y secundaria, a la educación superior. En este último nivel, algunas de las universidades más prestigiosas del mundo han apostado desde hace unos cuantos años por las TTD. Entre otras, podríamos citar las norteamericanas Massachussets Institute of Technology (MIT), Virginia Tech, Georgia Tech, Carnegie-Mellon, DePauw, Purdue, Michigan, California San Diego (UCSD) o la australiana Monash. Publicaciones de autores de estos centros destacan el impacto de estas tecnologías sobre el rendimiento académico, la motivación y la implicación de los estudiantes en su proceso de aprendizaje (Tront, 2007; Koile y Singer, 2008; Logan et al., 2009). En una investigación reciente (Mckenzie y Franke, 2009) se revisaron 144 trabajos sobre el uso educativo de las tabletas PC y se observó que, a nivel universitario, el 45\% de los mismos estaba relacionado con disciplinas de Informática. Mientras algunos de estos trabajos se referían a ámbitos multidisciplinares'(Anderson et al., 2004; Simon et al., 2004), otros estaban centrados en materias concretas talas como Introducción a la Informática (Koile y Singer, 2006), Estructuras de Datos (Calder et al., 2007), Teoría de Computadores (Mauch, 2008) o Arquitectura de Computadores (Almeida y Azevedo, 2009).

Aunque existe una abundante literatura que describe experiencias de uso de tabletas PC en distintos niveles educativos, resulta bastante más difícil encontrar contribuciones de cómo establecer estrategias que orienten a los docentes en la incorporación de estas tecnologías. A continuación, se repasan las más significativas. 
En (Hammond y Mock, 2008) se proporcionan algunas ideas prácticas de cómo incorporar tabletas PC no conectadas en red en el aula. Su esfuerzo se centra en cómo realizar presentaciones y en la adaptación de los materiales instructivos a esta tecnología. En (Evans, 2008) se da un nuevo paso y se aboga por desarrollar un mapa de ruta como el único camino para conseguir una implementación exitosa de la tecnología de tabletas PC. Este mapa de ruta debería articular primero los objetivos del proyecto; a continuación, debería identificar los pasos necesarios y los factores críticos requeridos en la implementación del proyecto y, por último, el plan de trabajo debería ayudar a todos los participantes a entender las decisiones tomadas antes de que se ponga en marcha. Las iniciativas anteriores permiten abordar las cuestiones generales sobre la implantación de experiencias con tabletas PC, pero no proporcionan directrices finales a los profesores.

Otros trabajos se centran más en el apoyo a los docentes para diseñar cursos con tabletas PC. En (Tront, 2007) se describe el programa implementado en el Virginia Tech College of Engineering desde el otoño de 2006 para facilitar las mejores prácticas pedagógicas que puedan suponer una mejora de aprendizaje. Entre otras muchas cosas, presenta algunas ideas y herramientas software para transformar el estilo de enseñanza, así como los materiales de instrucción correspondiente. En (Wolfman, 2005) se explora el potencial de la tecnología de tabletas PC para hacer frente a las necesidades de los instructores en la enseñanza de nivel universitario. En primer lugar describe los problemas más importantes que, en su opinión, deben afrontar los docentes con esta transformación (la participación de los estudiantes, la gestión del tiempo, la comprensión y adopción de nuevas perspectivas pedagógicas, la gestión de los grandes grupos). A continuación, describe las características tecnológicas que ofrece la tableta PC para hacer frente a estos problemas (crear anotaciones, colaborar a través de los dispositivos móviles conectados en red, la expresión personal, la posibilidad de almacenar los archivos generados...).

Si bien todos los trabajos previos ciertamente dan ideas para adaptar un modelo de enseñanza a la tecnología de tabletas PC, no hemos encontrado enfoques metodológicos para orientar estos procesos. En la sección siguiente se introduce nuestra propuesta de apoyo a los docentes que se plantean incorporar la tecnología de tabletas PC con el fin de crear entornos educativos más dinámicos.

\section{Uso de mapas conceptuales como soporte a los docentes}

La propuesta aquí presentada parte de la modelización del conocimiento tanto en lo que se refiere a los aspectos formativos como a los tecnológicos, referidos a un curso y entorno determinado. La representación de este conocimiento mediante mapas conceptuales ayuda a los profesores a entender mejor las posibilidades de las tabletas PC, al tiempo que facilita la búsqueda de aquellas características que mejor se adaptan a los requerimientos formativos planteados.

El primer paso en el proceso propuesto es desarrollar un mapa conceptual que represente el modelo instructivo del entorno de aprendizaje que va a ser objeto de intervención. Por tanto, una primera aproximación sería que los docentes implicados crearan, desde cero, un mapa conceptual que resumiera su enfoque formativo particular y que contemplara los distintos aspectos del curso o módulo a representar. Este mapa conceptual podría contemplar aspectos relacionados con los objetivos de aprendizaje, disciplina, perfil de los estudiantes, recursos didácticos, métodos docentes y actividades de aprendizaje, entre otros. 
Sin embargo, y con el fin de facilitar el procesamiento posterior de la información contenida en los mapas, se ha optado por construir un mapa instructivo suficientemente general que el profesor deberá adaptar a su situación particular. En este trabajo se utiliza, como ejemplo para la discusión posterior, un modelo concreto de enseñanza presencial basado en clase magistral, pues es todavía el enfoque más generalizado en nuestras titulaciones de ingeniería. La Figura 1 muestra el mapa conceptual general creado para representar dicho modelo de enseñanza. Este mapa introduce una estructura concreta donde los elementos situados en la parte superior del mismo, representan los conceptos que se han considerado clave en este tipo de cursos: contenidos, actividades, interacción y evaluación. Los niveles siguientes del mapa permiten detallar el enfoque seguido para configurar cada uno de esos conceptos. Evidentemente, el mapa instructivo introducido podría ciertamente ser mucho más complejo para así contemplar otras muchas estrategias docentes. Sin embargo, se ha decidido simplificarlo con el fin de facilitar la toma y análisis de datos.

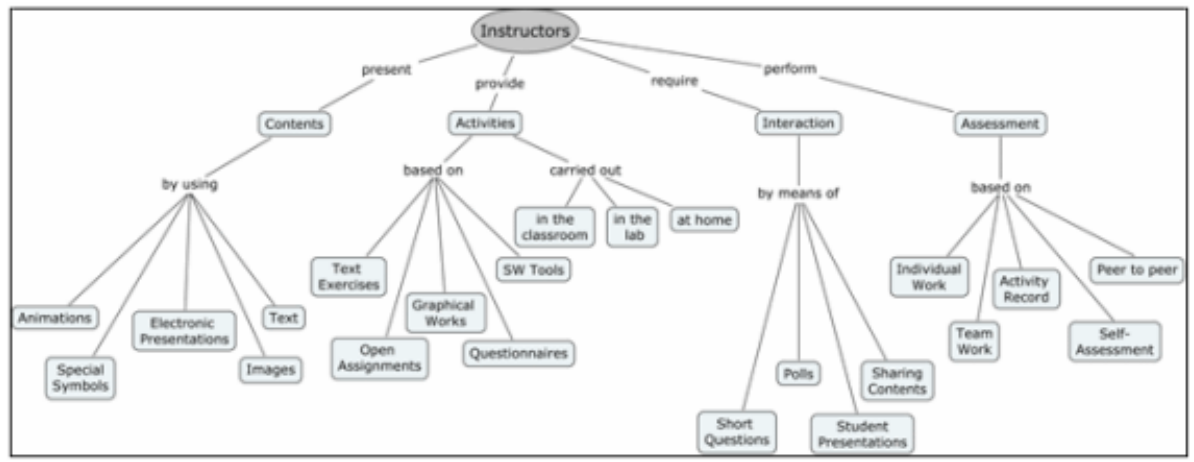

Figura 1: Mapa conceptual genérico para un enfoque basado en clase magistral

Los bloques definidos se pueden vincular fácilmente con los típicos problemas que surgen en este tipo de contextos, tales como la motivación de los alumnos, la selección y secuencia de contenidos, la configuración de actividades a realizar en el aula o formando parte del trabajo autónomo del alumno, o el establecimiento de procedimientos y criterios de evaluación, entre otros.

De forma similar, y en relación al dominio tecnológico, se ha desarrollado un mapa conceptual para modelizar el campo de las TTD. Este nuevo mapa utiliza como nodo raíz la tableta PC y, a partir de este concepto, trata de proporcionar una idea global sobre estas tecnologías: características y tipos de los dispositivos que las forman, sistemas operativos que les dan soporte, servicios que proporcionan, y herramientas software asociadas, entre otros aspectos. Dado el tamaño y complejidad del mapa generado, se ha optado por representar aquí dos submapas relacionados con dos de los conceptos más importantes incluidos en el mapa completo. En particular, las Figuras 2 y 3 representan, respectivamente, los conceptos tinta digital y servicios de las tabletas PC. 


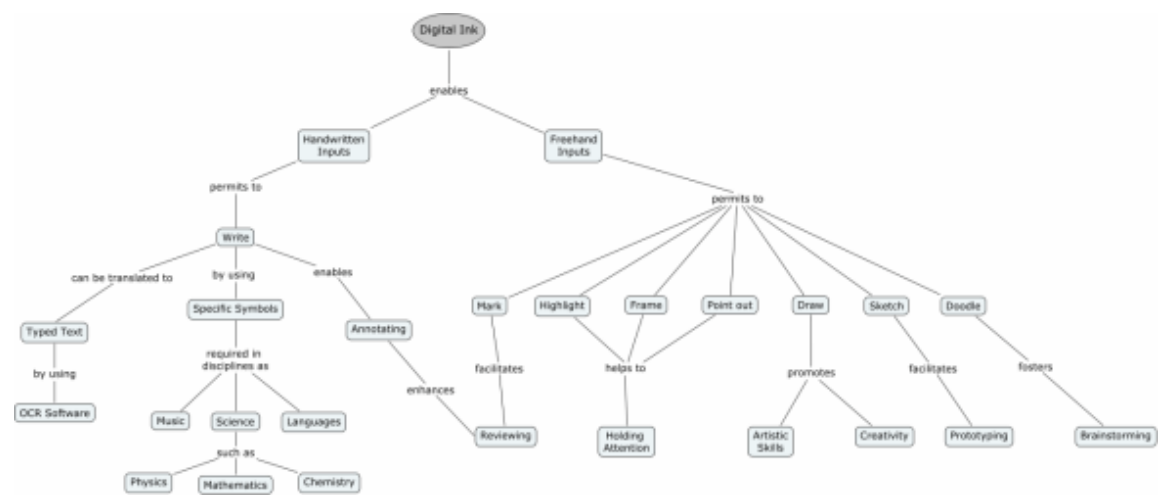

Figura 2: Submapa conceptual de la tinta digital y sus posibilidades formativas

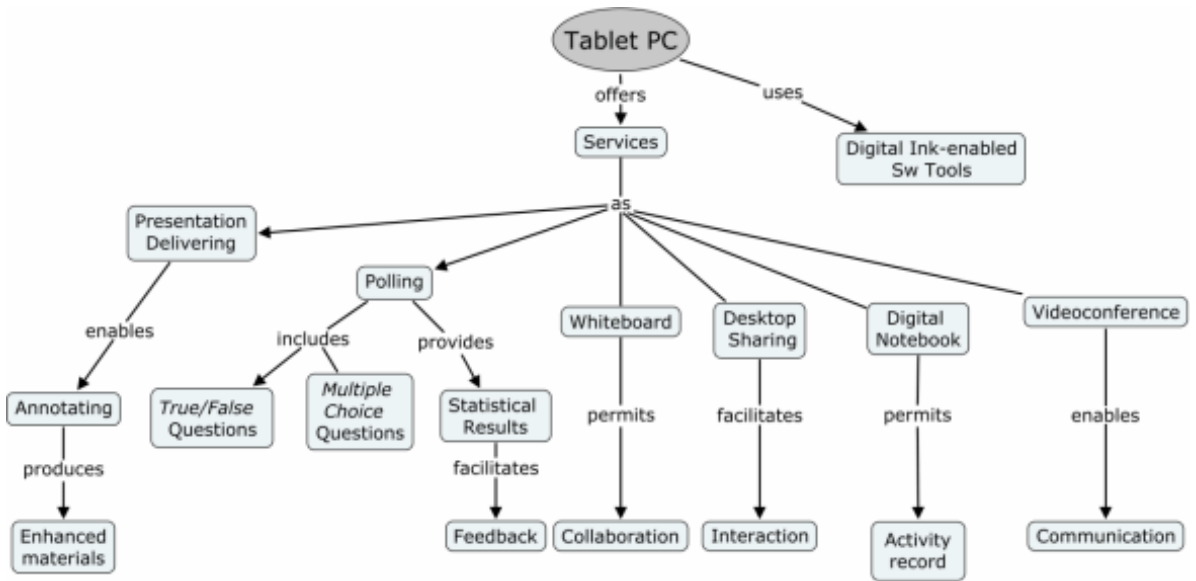

Figura 3: Submapa conceptual de los servicios de las tabletas PC

Resulta importante señalar que el submapa de tinta digital incorpora las relaciones entre la posibilidad de producir todo tipo de trazos directamente sobre la superficie de la pantalla, con las actividades formativas que se pueden potenciar tales como: anotación, revisión, lluvia de ideas (brainstorming), prototipado y diseño, entre otras. Del mismo modo, el submapa de la Figura 3 relaciona los servicios de las tabletas PC con los aspectos instructivos que se pueden fomentar, tales como la toma de notas, el trabajo colaborativo, la interacción, la comunicación o la realimentación, entre otros.

Una vez definidos los dominios instructivo y tecnológico (tableta PC) mediante los correspondientes mapas conceptuales, el siguiente paso es relacionarlos. Esto es, partiendo de los elementos que constituyen ambos mapas conceptuales (conceptos y relaciones), nuestra propuesta pretende realizar una inferencia de información que permita proporcionar a los docentes unas directrices de uso de las tecnologías, adaptadas al enfoque instructivo concreto. Este aspecto se aborda en la sección siguiente. 


\section{Generación de recomendaciones}

Con el fin de facilitar la adaptación del mapa conceptual general introducido (Figura 1) al escenario concreto de cada docente, los distintos conceptos de ese mapa general se han traducido en cuestiones que, ordenadas según la jerarquía del mapa de donde proceden, permiten elaborar un cuestionario completo. El análisis de las correspondientes respuestas permite recoger los requerimientos instructivos de cada uno de los profesores, al tiempo que genera un submapa donde aquellos conceptos que representan su enfoque instructivo particular aparecen resaltados. La Figura 4 representa un ejemplo de mapa conceptual creado a partir de las respuestas al cuestionario de un profesor que imparte un curso de informática industrial. En dicho mapa se han marcado en fondo más oscuro los conceptos correspondientes a su enfoque instructivo.

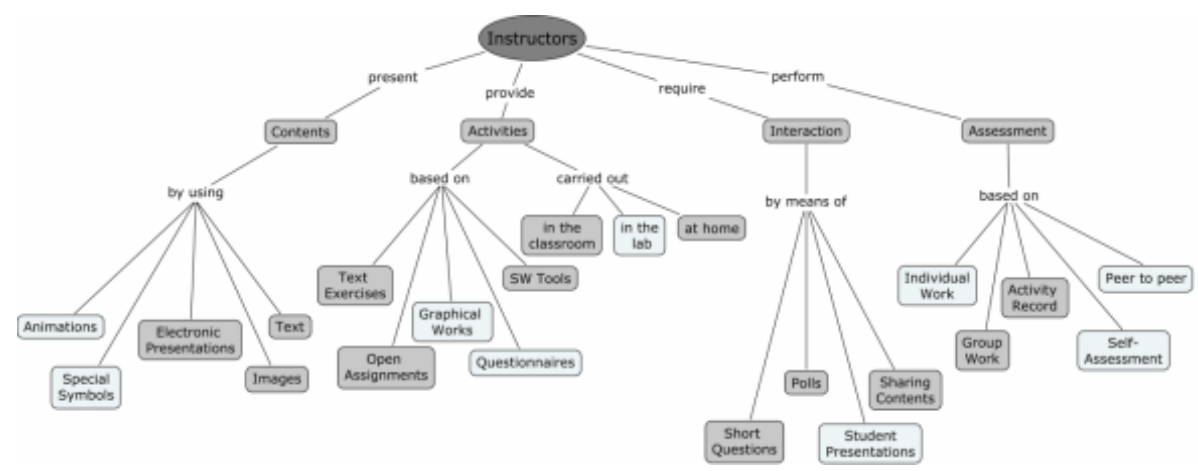

Figura 4: Selección de conceptos en el mapa conceptual instructivo

Así, podemos observar que en ese contexto concreto el profesor presenta sus contenidos haciendo uso de documentos de texto, imágenes y presentaciones electrónicas. Introduce actividades de aprendizaje en clase que son tareas a desarrollar, o se articulan alrededor de la lectura de textos o el uso de herramientas software; promueve la interacción con sus alumnos mediante preguntas cortas a la audiencia, la realización de sondeos y la compartición de contenidos entre grupos de trabajo y, finalmente, realiza la evaluación del estudiante mediante un registro de su actividad y la valoración del trabajo en equipo.

Una vez que el profesor ha especificado los aspectos instructivos de su curso, el proceso de correspondencia entre dominios se encarga de buscar los conceptos relacionados en el mapa del dominio tecnológico, en nuestro caso el de las tabletas PC, para así poder generar las recomendaciones de uso pertinentes. En particular, este proceso se centra en el submapa de servicios (Figura 3) y muy especialmente en sus conceptos terminales, ya que son los que recogen las posibilidades formativas de las tabletas PC. Siguiendo con el caso de informática industrial tomado como ejemplo, encontramos correspondencias con los conceptos siguientes (en fondo más oscuro): presentaciones mejoradas, interacción, colaboración, realimentación, registro de actividad, lo que nos lleva a recomendar un conjunto determinado de servicios (en fondo oscuro y sombreados), tal y como se resalta en la Figura 5. 


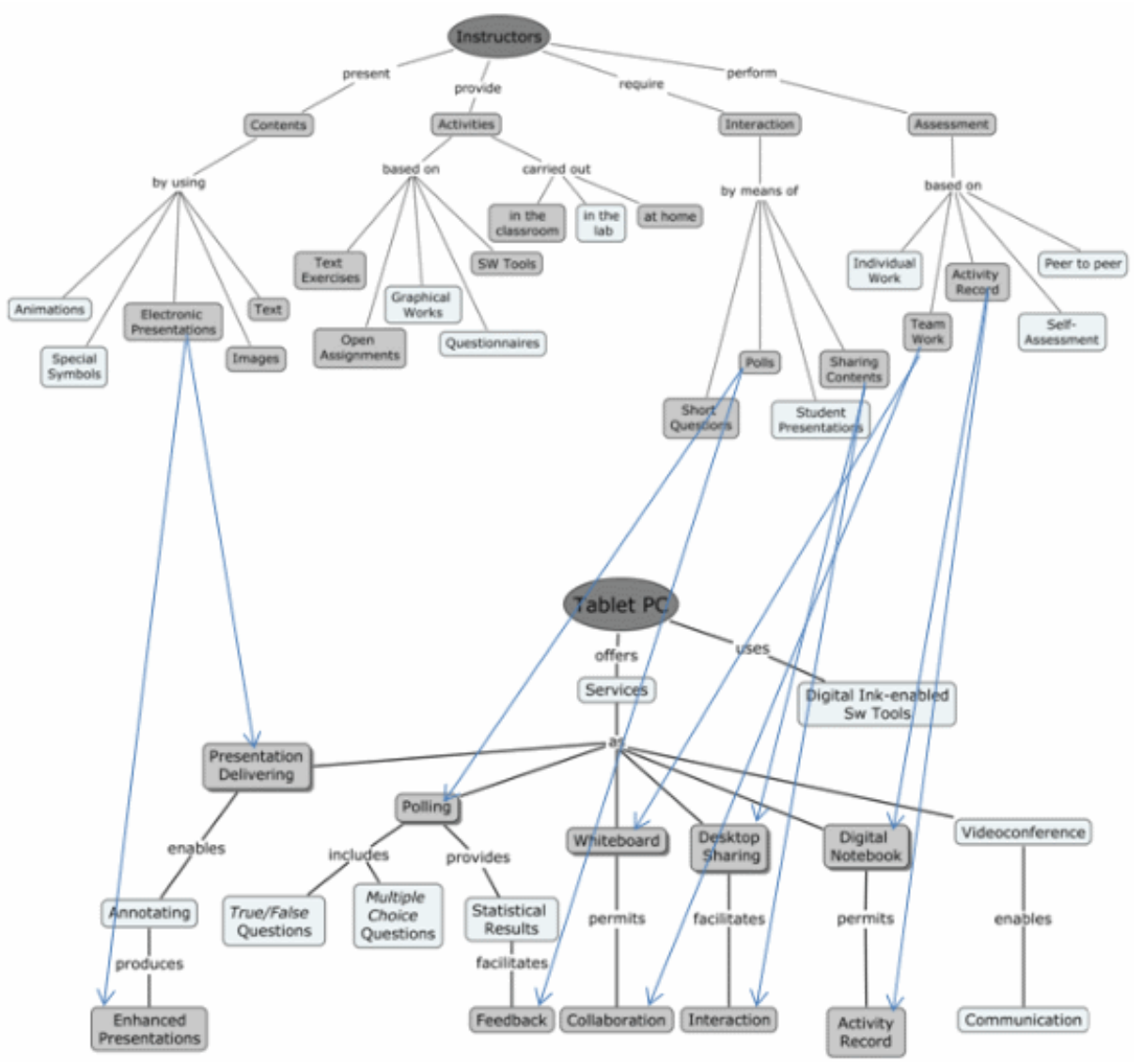

Figura 5: Correspondencias entre conceptos en los mapas instructivo y tecnológico

Finalmente se relaciona el submapa de servicios con el submapa de herramientas software con soporte para tinta digital, no mostrado aquí por su complejidad, donde se buscan ejemplos concretos de aplicaciones que sería recomendable utilizar.

Para el ejemplo descrito en este trabajo, todo el conjunto de relaciones se ha resumido en una tabla de recomendaciones (Tabla 1) que contiene tres columnas: la primera referida a las posibilidades concretas de uso de la tecnología, junto con ejemplos particulares de herramientas software; la segunda se refiere a las posibilidades formativas que introducen esas herramientas y, la tercera, los resultados que se esperan alcanzar en el proceso de enseñanzaaprendizaje. 
Tabla 1: Recomendaciones de uso de las Tabletas PC en el proceso formativo

\begin{tabular}{|c|c|c|}
\hline Uso de las tabletas PC & Posibilidades formativas & $\begin{array}{l}\text { Resultados en el proceso } \\
\text { de enseñanza-aprendiza- } \\
\text { je }\end{array}$ \\
\hline \begin{tabular}{|l|} 
Servicios de difusión de \\
presentaciones electrónicas \\
(presentation delivering), in- \\
cluidos en herramientas como \\
Classroom Presenter o Dy- \\
know, hacen uso de las fun- \\
cionalidades de la tinta digi- \\
tal y podrían ser utilizados \\
para:
\end{tabular} & $\begin{array}{l}\text { Aumentar la flexibilidad del pro- } \\
\text { fesor mientras da clase al poder } \\
\text { realizar anotaciones sobre las } \\
\text { presentaciones. } \\
\text { Facilitar la toma de notas de los } \\
\text { estudiantes sobre las propias } \\
\text { presentaciones. } \\
\text { Permitir a los estudiantes enviar } \\
\text { al profesor las actividades de clase } \\
\text { para su posterior revisión y dis- } \\
\text { cusión. } \\
\text { Permite archivar en formato elec- } \\
\text { trónico tanto las presentaciones } \\
\text { anotadas como las entregas de los } \\
\text { estudiantes. }\end{array}$ & $\begin{array}{l}\text { Materiales didácticos en- } \\
\text { riquecidos y dinámicos. } \\
\text { Mayor participación de los } \\
\text { estudiantes en el proceso. } \\
\text { Aprendizaje colaborativo } \\
\text { (los estudiantes pueden } \\
\text { aprender de las respuestas } \\
\text { de sus compañeros). } \\
\text { Comunicación mejorada } \\
\text { con otros compañeros y } \\
\text { con el profesor. } \\
\text { Realimentación oportuna } \\
\text { a los estudiantes y al } \\
\text { mismo profesor. }\end{array}$ \\
\hline $\begin{array}{l}\text { Servicio de escritorio com- } \\
\text { partido (desktop sharing), } \\
\text { como el proporcionado por } \\
\text { Yugma o Adobe Connect, } \\
\text { podría ser usado para: }\end{array}$ & $\begin{array}{l}\text { Atraer la atención de los estudi- } \\
\text { antes al introducir simulaciones } \\
\text { por computador como actividades } \\
\text { de aula. } \\
\text { Posibilitar que los estudiantes } \\
\text { asuman el papel del profesor para } \\
\text { realizar presentaciones al grupo. }\end{array}$ & $\begin{array}{l}\text { Aproximación realista de } \\
\text { la asignatura gracias al uso } \\
\text { de herramientas de simula- } \\
\text { ción, como PSpice, por } \\
\text { ejemplo. } \\
\text { Implicación y cambio de } \\
\text { rol de los estudiantes. }\end{array}$ \\
\hline \begin{tabular}{|l|} 
Servicios de sondeos \\
(polling), también incluidos \\
en herramientas como \\
Classroom Presenter o Dy- \\
know, podrían ser utilizados \\
para:
\end{tabular} & $\begin{array}{l}\text { Mejorar la consecución de los } \\
\text { logros de aprendizaje del estu- } \\
\text { diante. } \\
\text { Aumentar el conocimiento del } \\
\text { profesor sobre el grado de com- } \\
\text { prensión de los conceptos clave } \\
\text { expuestos en las lecciones. } \\
\text { Detectar malentendidos y pro- } \\
\text { poner refuerzos. }\end{array}$ & $\begin{array}{l}\text { Autoestima y confianza } \\
\text { del estudiante. } \\
\text { Realimentación oportuna } \\
\text { a los estudiantes y al } \\
\text { mismo profesor. }\end{array}$ \\
\hline $\begin{array}{l}\text { Servicio de pizarra virtual } \\
\text { (whiteboard), incorporado en } \\
\text { herramientas como Vyew.com } \\
\text { o Echalk, podría utilizarse } \\
\text { para: }\end{array}$ & $\begin{array}{l}\text { Facilitar la realización de trabajos } \\
\text { en grupo compartiendo un espacio } \\
\text { virtual. }\end{array}$ & Aprendizaje colaborativo. \\
\hline
\end{tabular}




\begin{tabular}{|c|c|c|}
\hline $\begin{array}{l}\text { Servicios de cuaderno digit- } \\
\text { al (digital notebook), } \\
\text { como el ofrecido por } M S \\
\text { Office OneNote, podría ser } \\
\text { usado para: }\end{array}$ & $\begin{array}{l}\text { Recoger todas las actividades rela- } \\
\text { cionadas a lo largo del curso (a } \\
\text { modo de portafolio). } \\
\text { Revisar las tareas de los estudian- } \\
\text { tes y proporcionarles comentarios } \\
\text { y sugerencias de aprendizaje. }\end{array}$ & $\begin{array}{l}\text { Implicación del estudiante. } \\
\text { Seguimiento de la actividad } \\
\text { del estudiante (evaluación } \\
\text { continua). }\end{array}$ \\
\hline
\end{tabular}

\section{Validación de la propuesta}

Para validar la propuesta descrita, durante los cursos académicos 2010-11 y 2011-12 se han llevado a cabo una serie de talleres donde se presentaba a los docentes las posibilidades de las TTD y, al mismo tiempo, experimentaban con tabletas PC y otros dispositivos de tinta digital, en un aula configurada para tal propósito. Más de setenta profesores vinculados a estudios de ingeniería han participado en los mismos.

Antes de comenzar los talleres, se proporcionaba a los asistentes un cuestionario (precuestionario) con el fin de obtener su modelo instructivo particular. Tal y como se comentó anteriormente, para ello se hizo uso del conjunto de preguntas generado a partir del mapa conceptual genérico de la Figura 1. Partiendo de las posibilidades instructivas de las TTD contempladas en el mapa conceptual tecnológico y de la propia experiencia en la aplicación de dichas tecnologías a distintas disciplinas, se ha establecido una lista de buenas prácticas de uso de las mismas.

La Figura 6 resume las contestaciones a los pre-cuestionarios de los asistentes a los seminarios celebrados durante esos dos cursos. En concreto, se muestran únicamente los porcentajes obtenidos en las preguntas referidas a aquellos aspectos del modelo instructivo general, donde las TTD han demostrado mayores posibilidades. Destaca el elevado número de profesores que utiliza presentaciones electrónicas en las clases magistrales (92\%); se apoya de elementos gráficos como diagramas, figuras o esquemas, para presentar contenidos (80\%); o incorpora algún elemento de evaluación del estudiante en el aula (68\%). También son frecuentes los docentes que plantean actividades que hacen uso de elementos gráficos $(73 \%)$ o ejercicios de respuesta abierta $(70 \%)$. Todas estas estrategias pueden considerarse comunes en buena parte de nuestras titulaciones de ingeniería.

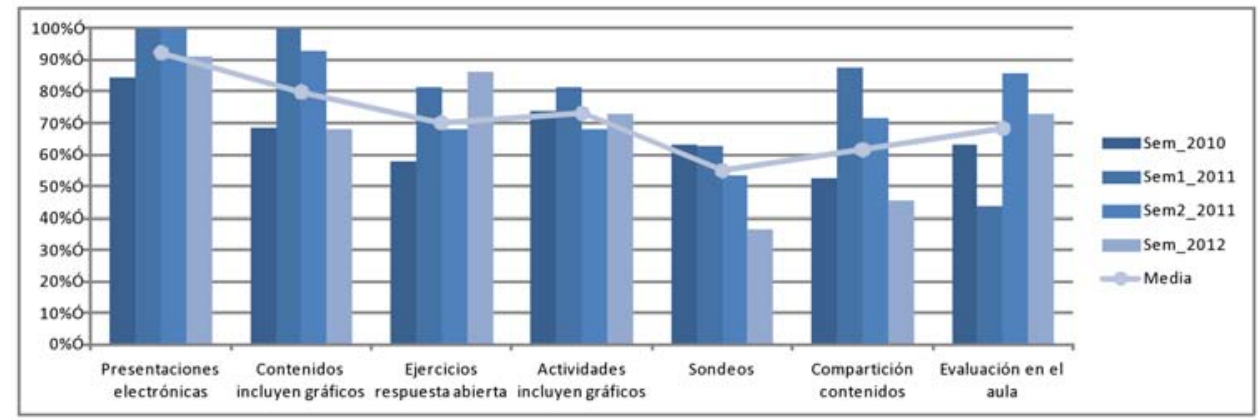

Figura 6: Resumen de resultados del pre-cuestionario 
La definición de buenas prácticas citada anteriormente ha servido también para elaborar un cuestionario sobre las posibilidades de las TTD que serán presentadas a los profesores al finalizar los talleres (post-cuestionario). De este modo, y tras experimentar con las tecnologías, los participantes en los talleres valoran la idoneidad de las posibilidades instructivas de estos dispositivos en sus contextos particulares.

La Figura 7 resume las contestaciones de los profesores al post-cuestionario. En primer lugar, llama la atención los elevados porcentajes obtenidos en prácticamente todas las dimensiones analizadas (por encima del 79\%) que, según los profesores participantes en los talleres, confirman el potencial de las TTD para mejorar esos aspectos. En particular, destacan la facilidad para realizar sondeos (94\%), exponer ideas de forma gráfica $(91 \%)$, realizar tareas que requieran elementos gráficos $(88 \%)$ o evaluar a los estudiantes en clase (83\%). Trabajos futuros en esta misma línea tratarán de reforzar los resultados obtenidos con la participación de más profesores en nuevos talleres y la aplicación de las TTD a nuevas áreas de conocimiento.

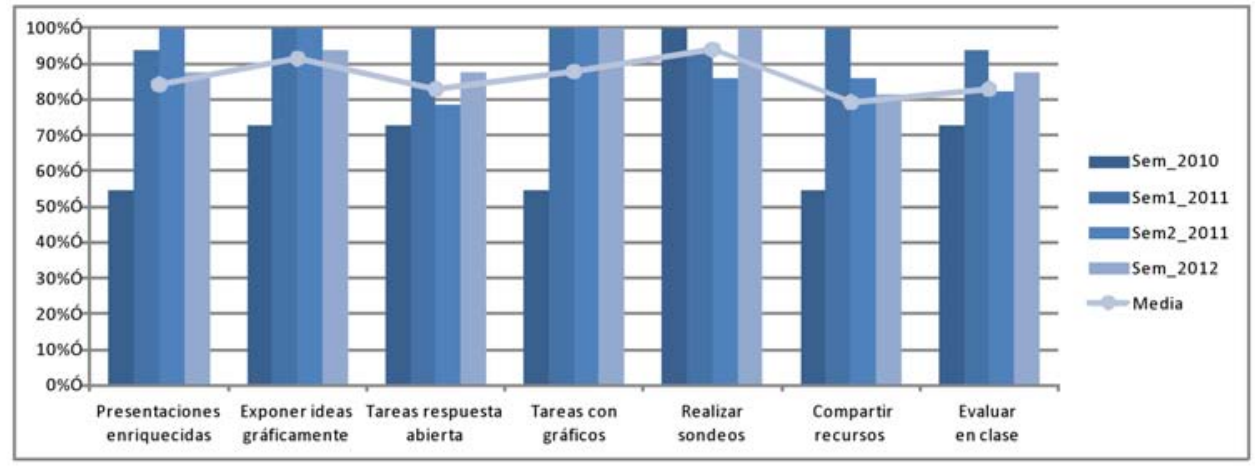

Figura 7: Resumen de resultados del post-cuestionario

\section{Conclusiones}

En este trabajo se ha presentado una propuesta para ayudar a los docentes que se plantean incorporar TTD para crear unos entornos de aprendizaje más dinámicos. La propuesta plantea el uso de mapas conceptuales para modelizar tanto los aspectos instructivos de un entorno concreto como las posibilidades formativas de las tecnologías de tinta digital. La correspondencia entre ambos mapas conceptuales permite generar una serie de recomendaciones que ayudan al docente en la incorporación de las tecnologías a dicho entorno. Para validar el modelo se han desarrollado una serie de talleres que confirman el potencial de las tecnologías en el ámbito de las ingenierías y permiten la mejora continua de la propuesta. 


\section{Referencias}

Almeida, P. y Azevedo, R. (2009). "Active Learning and Screencasting with Tablet PCs: A Detailed Evaluation”. En: D.A. Berque, L. M. Konkle y R.H. Reed (eds.), The Impact of Tablet PCs and Pen-based Technology on Education. New Horizons (pp. 3-11), West Lafayette, Indiana: Purdue University Press.

Anderson, R., Anderson R., Simon, B., Wolfman, S., VanDeGrift, T. y Yasuhara, K. (2004). "Experiences with a Tablet PC Based Lecture Presentation System in Computer Science Courses". En: Proceedings of SIGCSE '04, 56-60.

Buendia, F. (2011). "Supporting the Generation of Guidelines for Online Courses," Journal of eLearning and Knowledge Society, 7(3), 51-61.

Calder, M., Cohen, R.F., Lanzoni, J., Landry, N., y Skaff J. (2007). "Teaching data structures to students who are blind". SIGCSE Bull. 39, 3, 87-90.

Chickering, A. W. y Ehrmann S.C. (1996). "Implementing the Seven Principles: Technology as Lever," AAHE (American Association of Higher Education) Bulletin, 49(2), 3-6.

Chickering, A. W. y Gamson, Z.F. (1987). "Seven Principles for Good Practice in Undergraduate Education", AAHE (American Association of Higher Education) Bulletin, 39(7), 3-7.

Deal, A. (2007). "Classroom Response Systems, A Teaching with Technology White Paper," Office of Technology for Education, Carnegie Mellon University, (http://www.cmu.edu/teaching/resources/PublicationsArchives/StudiesWhitepapers/ClassroomResponse_Nov07.pdf)

Ehrmann, S. C., (2008). "Seven Principles. Collection of Ideas for Teaching and Learning with Technology" (http://www.tltgroup.org/seven/Library_TOC.htm).

Evans, E. J. (2008). "Deploying Tablet PC in your school". En: M. van Mantgem (ed.), Tablet PC in K-12 Education (pp. 137-151), Eugene, OR: International Society for Technology in Education (ISTE).

Fundación de la Innovación Bankinter (2011). "La educación del siglo XXI. Una apuesta de futuro". (http://www.fundacionbankinter.org/system/documents/8498/original/FTFXVI_Educacion_FINAL.pdf).

Fundación Telefónica (2011). "Universidad 2020: Papel de las TIC en el nuevo entorno socio-económico". Ariel.

Hammond, T. y Mock, K. (2008). “Bringing Single-User Settings to Life”. En: M. van Mantgem (ed.), Tablet PC in K-12 Education (pp. 87-112), Eugene, OR: International Society for Technology in Education (ISTE).

Koile, K. y Singer, D. (2006). "Improving learning in CS1 via tablet-PC-based in-class assessment". En: Proceedings of the second international workshop on Computing education research (ICER '06). New York, NY: ACM, 119-126.

Koile, K. y Singer, D. (2008). "Assessing the Impact of a Tablet-PC-based Classroom Interaction System”. En: R.H. Reed, D.A. Berque, y J.C. Prey (eds.), The Impact of Tablet PCs and Pen-based Technology on Education. Evidence and Outcomes (pp. 73-80), West Lafayette, Indiana: Purdue University Press.

Logan, M., Bailey, N., Franke, K. y Sanson, G. (2009). "Patterns of Tablet PC Use across Multiple Learning Domains: A Comparison Program”. En: D.A. Berque, L. M. Konkle y R.H. Reed (eds.), The Impact of Tablet PCs and Pen-based Technology on Education. New Horizons (pp. 83-92), West Lafayette, Indiana: Purdue University Press.

Mauch, H. (2008). “Tablet PCs: Impacting student learning in Computer Theory instruction”. En: R.H. Reed, D.A. Berque, y J.C. Prey (eds.), The Impact of Tablet PCs and Pen-based Technology on Education. Evidence and Outcomes (pp. 95-102), West Lafayette, Indiana: Purdue University Press.

Mckenzie, W. y Franke, K. (2009). “Active, Constructive, Interactive: How are Tablet PCs Transforming the Learning Experience in Higher Education?", Presentation at the 2009 Australasian Tablets in Education Conference, Monash University, Melbourne, 3-4 December. (http://www.monash.edu/eeducation/assets/documents/atiec/2009atiec-wendymckenzie.pdf) 
Novak, J.D., (1998). Learning, creating, and using knowledge: Concept maps as facilitative tools in schools and corporations. Mahwah, NJ: Lawrence Erlbaum Associates.

Simon, B., Anderson, R., Hoyer, C., y Su, J. (2004). "Preliminary experiences with a tablet PC based system to support active learning in computer science courses". En: Proceedings of the 9th annual SIGCSE Conference on Innovation and Technology in Computer Science Education (ITiCSE '04). New York, NY: ACM, 213-217.

Sneller, J. (2007). "The Tablet PC classroom: Erasing borders, stimulating activity, enhancing communication". En: 37th ASEE/IEEE Frontiers in Education Conference, IEEE.

Tront, J.G. (2007). "Facilitating Pedagogical Practices through a Large-Scale Tablet PC Deployment". Computer, 40(9), 62-68.

Wolfman, S. (2005), “Teacher's group notes”, En: 2005 Tablet PC in Higher Education Workshop, Jul 2005. Seattle, WA (http://www.cs.washington.edu/homes/anderson/tpc/documents/wolf.html).

\section{Sobre los Autores}

Jose-Vicente Benlloch-Dualde: José-Vicente Benlloch-Dualde was born in Valencia (Spain). He earned a MSc. in Physics from Universitat de València in 1986. Currently, he is Senior Lecturer with tenure at the Escuela Técnica Superior de Ingeniería Informática in the Universitat Politècnica de València, where teaches Computer Technology and several elective courses about Multimedia Systems. His research interests relate to technology-enhanced learning and pen-based technology. He earned a HP Technology for Teaching Grant Initiative, Transforming Teaching and Learning through Technology in 2008. He is member of IEEE and EAEEIE among other international associations.

\section{Félix Buendía: -}

\section{Juan-Carlos Cano: -}

Sara Blanc: Sara Blanc Clavero se licenció en Ingeniería Informática en el año 1998 en la Universidad Politécnica de Valencia, en España. En el año 2004 se doctoró por la misma universidad en la que actualmente imparte docencia en el Dpto. de Informática de Sistemas y Computadores. Actualmente, sus intereses se dividen entre docencia e investigación. En docencia, imparte distintos grupos en materias relacionadas con el grado de Ingeniería Informática, Ingeniería de Telecomunicaciones e Ingeniería Industrial. En investigación, sus últimos trabajos se centran en el desarrollo de redes de sensores subacuáticas.

Lenin Lemus: - 